\title{
FINITE STRAIN SOLUTIONS FOR A COMPRESSIBLE ELASTIC SOLID
}

\author{
BY \\ M. M. CARROLL (Rice University, Houston, Texas) \\ AND \\ C. O. HORGAN (University of Virginia, Charlottesville, Virginia)
}

\begin{abstract}
Several closed form finite strain equilibrium solutions are presented for a special compressible isotropic elastic material which was proposed as a model for foam rubber by Blatz and Ko. These solutions include bending of a cylindrical sector into another sector or a rectangular block, bending of a block into a sector, expansion, compaction or eversion of cylinders or spheres, and torsion and extension of circular cylinders or tubes.
\end{abstract}

1. Introduction. Ericksen has examined the problems of finding all of the deformations which can be supported, in the absence of body force, in all homogeneous, isotropic, incompressible elastic solids [1], or in all homogeneous, isotropic, compressible elastic solids [2]. The first category consists of all isochoric homogeneous deformations and five families of nonhomogeneous deformations, the so-called controllable or universal deformations ([1] and Singh and Pipkin [3]). The second category consists of homogeneous deformations only, i.e., there is no nonhomogeneous finite deformation which can be supported in every compressible isotropic elastic solid material without applying a body force.

This latter result implies that nonhomogeneous deformations can be discussed, for compressible solids, only in the context of a particular strain energy function, or class of strain energy functions. Currie and Hayes [4] have suggested that Ericksen's results have had an unduly inhibiting effect on the study of nonhomogeneous finite deformations. For compressible solids, in particular, the analysis of such deformations is usually very complicated even for simple forms of the strain energy function and very few closed form solutions have been obtained.

John [5] introduced the class of harmonic materials, for which the problem of finite plane strain simplifies considerably, and solutions of such problems have been presented by several authors [6-10]. Abeyaratne and Horgan [11] obtained an exact solution of the problem of pressurization of a hollow sphere of harmonic material; see also Ogden [10]. Wheeler [12] has examined the deformation of a harmonic material containing an ellipsoidal cavity. Chung, Horgan, and Abeyaratne [13] obtained 
exact solutions of the problems of cylindrical or spherical expansion or compaction for a material with a special strain energy function proposed by Blatz and Ko [14] to model the nonlinear response of foam rubber; see also Beatty [15]. Carroll [16] recently presented several exact solutions for three classes of materials, one of which is the class of harmonic materials. Haughton [17] has discussed inflation of thick-walled elastic spherical shells composed of compressible materials, including harmonic materials. Horgan [18] has recently described how the governing equilibrium equation for axisymmetric deformation of compressible materials in two and three dimensions can be reduced to a pair of first-order ordinary differential equations, leading to exact solutions of the equilibrium equation for particular materials.

In the present paper, exact solutions of several problems are found for the special strain energy proposed by Blatz and Ko [14]. These solutions are obtained by the semi-inverse method, and each of the deformations is a nonisochoric generalization of a deformation which is controllable for homogeneous, isotropic, incompressible elastic solids. Some of the solutions are quite simple (for example, the Blatz-Ko strain energy admits pure torsion*) and some are quite complicated (for example, the radial deformation solutions of Chung, Horgan, and Abeyaratne [13]). Although the Blatz-Ko strain energy is a very special one (even its response to infinitesimal deformations is special, with value $\frac{1}{4}$ for Poisson's ratio) it has been proposed as a model of compressible nonlinear elastic response for foam-rubber-like material. So, the solutions presented here are a worthwhile addition to the small set of closed form solutions in nonlinear elastostatics.

2. Basic equations. The strain energy function for a compressible isotropic elastic solid may be written as a function of the principal strain invariants

$$
W=W\left(I_{1}, I_{2}, I_{3}\right),
$$

with

$$
\begin{aligned}
& I_{1}=\operatorname{tr} \mathbf{B}=\lambda_{1}^{2}+\lambda_{2}^{2}+\lambda_{3}^{2}, \\
& I_{2}=\operatorname{tr} \mathbf{B}^{*}=\lambda_{2}^{2} \lambda_{3}^{2}+\lambda_{3}^{2} \lambda_{1}^{2}+\lambda_{1}^{2} \lambda_{2}^{2}, \\
& I_{3}=\operatorname{det} \mathbf{B}=\lambda_{1}^{2} \lambda_{2}^{2} \lambda_{3}^{2} .
\end{aligned}
$$

Here $\mathbf{B}=\mathbf{F F}^{\mathbf{T}}$, where $\mathbf{F}$ is the deformation gradient tensor, $\mathbf{B}^{*}=(\operatorname{det} \mathbf{B}) \mathbf{B}^{-1}$ is the adjoint of $\mathbf{B}$ and $\lambda_{1}, \lambda_{2}$, and $\lambda_{3}$ are the principal stretches. The corresponding response equation for the Cauchy stress tensor $\mathbf{T}$ is

$$
\mathbf{T}=2 I_{3}^{-1 / 2}\left[\left(I_{2} \frac{\partial W}{\partial I_{2}}+I_{3} \frac{\partial W}{\partial I_{3}}\right) \mathbf{1}+\frac{\partial W}{\partial I_{1}} \mathbf{B}-I_{3} \frac{\partial W}{\partial I_{2}} \mathbf{B}^{-1}\right]
$$

where $\mathbf{1}$ is the unit tensor.

The present paper treats a very special strain energy function

$$
W=\frac{1}{2} \mu\left(\frac{I_{2}}{I_{3}}+2 I_{3}^{1 / 2}-5\right) \text {, }
$$

\footnotetext{
${ }^{*}$ Currie and Hayes [4] identified the class of compressible isotropic elastic solids which admit pure torsion.
} 
for which the stress response equation (2.3) takes the simple form

$$
\mathbf{T}=\mu\left(\mathbf{1}-I_{3}^{-1 / 2} \mathbf{B}^{-1}\right) .
$$

The strain energy function (2.4) was proposed by Blatz and Ko [14] as a model for the nonlinear response of foam rubber. The response for a variety of stress and strain states has been examined by Knowles and Sternberg [19]. Explicit solutions of the problems of finite radial expansion or compaction of hollow spheres or cylinders were presented in a recent paper by Chung, Horgan, and Abeyaratne [13].

The equation of equilibrium, in the absence of body force, is

$$
\operatorname{div} \mathbf{T}=\mathbf{0} .
$$

The component form of this equation, in terms of the physical components of $T$ in a cylindrical polar system, is

$$
\begin{gathered}
\frac{\partial T_{r r}}{\partial r}+\frac{1}{r} \frac{\partial T_{r \theta}}{\partial \theta}+\frac{\partial T_{r z}}{\partial z}+\frac{1}{r}\left(T_{r r}-T_{\theta \theta}\right)=0, \\
\frac{\partial T_{\theta r}}{\partial r}+\frac{1}{r} \frac{\partial T_{\theta \theta}}{\partial \theta}+\frac{\partial T_{\theta z}}{\partial z}+\frac{2}{r} T_{\theta r}=0, \\
\frac{\partial T_{z r}}{\partial r}+\frac{1}{r} \frac{\partial T_{z \theta}}{\partial \theta}+\frac{\partial T_{z z}}{\partial z}+\frac{1}{r} T_{z r}=0 .
\end{gathered}
$$

The corresponding equations for a spherical polar system are

$$
\begin{gathered}
\frac{\partial T_{r r}}{\partial r}+\frac{1}{r} \frac{\partial T_{r \theta}}{\partial \theta}+\frac{1}{r \sin \theta} \frac{\partial T_{r \phi}}{\partial \phi}+\frac{1}{r}\left(2 T_{r r}-T_{\theta \theta}-T_{\phi \phi}+\cot \theta T_{r \theta}\right)=0, \\
\frac{\partial T_{\theta r}}{\partial r}+\frac{1}{r} \frac{\partial T_{\theta \theta}}{\partial \theta}+\frac{1}{r \sin \theta} \frac{\partial T_{\theta \phi}}{\partial \phi}+\frac{1}{r}\left[3 T_{r \theta}+\cot \theta\left(T_{\theta \theta}-T_{\phi \phi}\right)\right]=0, \\
\frac{\partial T_{\phi r}}{\partial r}+\frac{1}{r} \frac{\partial T_{\phi \theta}}{\partial \theta}+\frac{1}{r \sin \theta} \frac{\partial T_{\phi \phi}}{\partial \phi}+\frac{1}{r}\left(3 T_{r \phi}+2 \cot \theta T_{\theta \phi}\right)=0 .
\end{gathered}
$$

3. Straightening of a cylindrical sector. A deformation with coordinate representation

$$
x=\hat{x}(R), \quad y=B \Theta, \quad z=\lambda Z,
$$

with $\frac{d x}{d R}>0, B>0$, and $\lambda>0$, describes straightening of a cylindrical sector into a rectangular block. Here $(R, \Theta, Z)$ are cylindrical polar coordinates of a particle before deformation and $(x, y, z)$ are its rectangular Cartesian coordinates after deformation.

The deformation gradient tensor $\mathbf{F}$, given by

$$
\mathbf{F}=\frac{d x}{d R} \mathbf{e}_{x} \otimes \mathbf{e}_{n}+\frac{B}{R} \mathbf{e}_{y} \otimes \mathbf{e}_{\Theta}+\lambda \mathbf{e}_{z} \otimes \mathbf{e}_{Z},
$$

admits a polar decomposition $\mathbf{F}=\mathbf{V R}$, with rotation tensor $\mathbf{R}$ and stretch tensor $\mathbf{V}$ given by

$$
\mathbf{R}=\mathbf{e}_{x} \otimes \mathbf{e}_{R}+\mathbf{e}_{y} \otimes \mathbf{e}_{\Theta}+\mathbf{e}_{z} \otimes \mathbf{e}_{Z}
$$

and

$$
\mathbf{V}=\frac{d x}{d R} \mathbf{e}_{x} \otimes \mathbf{e}_{x}+\frac{B}{R} \mathbf{e}_{y} \otimes \mathbf{e}_{y}+\lambda \mathbf{e}_{z} \otimes \mathbf{e}_{z} .
$$


Substitution from Eq. (3.4), with

$$
I_{3}^{1 / 2}=\operatorname{det} \mathbf{V}, \quad \mathbf{B}=\mathbf{V}^{2}
$$

in the stress response equation (2.5), gives the principal stresses as

$$
\begin{gathered}
T_{x x}=\mu\left(1-\frac{R}{\lambda B}\left(\frac{d R}{d x}\right)^{3}\right), \quad T_{y y}=\mu\left(1-\frac{R^{3}}{\lambda B^{3}} \frac{d R}{d x}\right), \\
T_{z z}=\mu\left(1-\frac{R}{\lambda^{3} B} \frac{d R}{d x}\right) .
\end{gathered}
$$

The equation of equilibrium (2.6) reduces to

$$
\frac{d T_{x x}}{d x}=0
$$

and, if faces $x=$ constant are to be free of traction, this integrates to

$$
T_{x x}=0
$$

or

$$
\frac{d R}{d x}=\left(\frac{\lambda B}{R}\right)^{1 / 3}
$$

Thus, the deformation (3.1) has the form

$$
x=\frac{3}{4} \frac{1}{(\lambda B)^{1 / 3}} R^{4 / 3}, \quad y=B \Theta, \quad z=\lambda Z .
$$

The constant of integration, which represents a rigid body translation, has been set to zero.

The conditions for vanishing resultant normal forces on the faces $y=$ constant and $z=$ constant are

$$
\begin{aligned}
\int_{x_{1}}^{x_{2}} T_{y y} d x & =\mu \int_{R_{1}}^{R_{2}}\left(1-\frac{R^{3}}{\lambda B^{3}} \frac{d R}{d x}\right) \frac{d x}{d R} d R \\
& =\mu \int_{R_{1}}^{R_{2}}\left(\left(\frac{R}{\lambda B}\right)^{1 / 3}-\frac{R^{3}}{\lambda B^{3}}\right) d R=0
\end{aligned}
$$

and

$$
\begin{aligned}
\int_{x_{1}}^{x_{2}} T_{z z} d x & =\mu \int_{R_{1}}^{R_{2}}\left(1-\frac{R}{\lambda^{3} B} \frac{d R}{d x}\right) \frac{d x}{d R} d R \\
& =\mu \int_{R_{1}}^{R_{2}}\left(\left(\frac{R}{\lambda B}\right)^{1 / 3}-\frac{R}{\lambda^{3} B}\right) d R=0 .
\end{aligned}
$$

Integration gives

$$
\frac{3\left(R_{2}^{4 / 3}-R_{1}^{4 / 3}\right)}{4(\lambda B)^{1 / 3}}=\frac{R_{2}^{4}-R_{1}^{4}}{4 \lambda B^{3}}=\frac{R_{2}^{2}-R_{1}^{2}}{2 \lambda^{3} B}
$$

so that the constants $B$ and $\lambda$ are given by

$$
B^{2}=\frac{1}{2} \lambda^{2}\left(R_{1}^{2}+R_{2}^{2}\right), \quad \lambda^{10}=\frac{16\left(R_{2}^{2}-R_{1}^{2}\right)^{3}}{27\left(R_{2}^{2}+R_{1}^{2}\right)\left(R_{2}^{4 / 3}-R_{1}^{4 / 3}\right)^{3}}
$$


The resultant end couple $M$ needed to hold the straightened sector is given by

$$
M=\lambda L \int_{R_{1}}^{R_{2}} T_{y y} \frac{d x}{d R} d R
$$

where $L$ is the length of the sector, in the $Z$-direction, before deformation.

4. Bending of a rectangular block. A deformation with coordinate representation

$$
r=r(X), \quad \theta=B Y, \quad z=\lambda Z,
$$

with $\frac{d r}{d X}>0, B>0$, and $\lambda>0$, describes bending of a rectangular block into a cylindrical sector. Here $(X, Y, Z)$ and $(r, \theta, z)$ are rectangular Cartesian coordinates before deformation and cylindrical polar coordinates after deformation.

The deformation gradient tensor $\mathbf{F}$, given by

$$
\mathbf{F}=\frac{d r}{d X} \mathbf{e}_{r} \otimes \mathbf{e}_{X}+B r \mathbf{e}_{\theta} \otimes \mathbf{e}_{Y}+\lambda \mathbf{e}_{z} \otimes \mathbf{e}_{Z},
$$

admits a polar decomposition $\mathbf{F}=\mathbf{V R}$, with

$$
\mathbf{R}=\mathbf{e}_{r} \otimes \mathbf{e}_{X}+\mathbf{e}_{\theta} \otimes \mathbf{e}_{Y}+\mathbf{e}_{z} \otimes \mathbf{e}_{Z}
$$

and

$$
\mathbf{V}=\frac{d r}{d X} \mathbf{e}_{r} \otimes \mathbf{e}_{r}+B r \mathbf{e}_{\theta} \otimes \mathbf{e}_{\theta}+\lambda \mathbf{e}_{z} \otimes \mathbf{e}_{z}
$$

Substitution from Eq. (4.4) in the stress response equation (2.5) gives the principal stresses as

$$
\begin{gathered}
T_{r r}=\mu\left(1-\frac{1}{\lambda B r}\left(\frac{d X}{d r}\right)^{3}\right), \quad T_{\theta \theta}=\mu\left(1-\frac{1}{\lambda B^{3} r^{3}} \frac{d X}{d r}\right), \\
T_{z z}=\mu\left(1-\frac{1}{\lambda^{3} B r} \frac{d X}{d r}\right) .
\end{gathered}
$$

The equations of equilibrium (2.7) reduce to the radial equation

$$
\frac{d T_{r r}}{d r}+\frac{1}{r}\left(T_{r r}-T_{\theta \theta}\right)=0
$$

Substitution from Eqs. (4.5) 1,2 in this equation leads to

$$
\frac{d X}{d r} \frac{d^{2} X}{d r^{2}}=\frac{1}{3 B^{2} r^{3}}
$$

A first integration gives

$$
\frac{d X}{d r}=\frac{\sqrt{\alpha r^{2}-1}}{B r \sqrt{3}}
$$

where $\alpha>0$ is a constant of integration. A second integration gives

$$
X=\frac{1}{B \sqrt{3}}\left(\sqrt{\alpha r^{2}-1}-\sec ^{-1} r \sqrt{\alpha}+\beta\right) .
$$

Observe that this does not depend on the axial stretch $\lambda$. The stresses are obtained by substitution from Eq. (4.8) in Eqs. (4.5) and boundary conditions determine the unknowns $B, \lambda, \alpha$, and $\beta$ in the usual manner. 
5. Bending of a cylindrical sector. A deformation with coordinate representation

$$
r=\hat{r}(R), \quad \theta=B \Theta, \quad z=\lambda Z,
$$

with $\frac{d r}{d R}>0, B>0$, and $\lambda>0$, describes bending and axial stretching of a cylindrical sector. Here $(R, \Theta, Z)$ and $(r, \theta, z)$ are cylindrical polar coordinates of a particle before and after deformation. When $B=1$ and $\lambda=1$, the deformation reduces to the case of radial expansion or compaction, treated by Chung, Horgan, and Abeyaratne [13].

The deformation gradient tensor

$$
\mathbf{F}=\frac{d r}{d R} \mathbf{e}_{r} \otimes \mathbf{e}_{R}+\frac{B r}{R} \mathbf{e}_{\theta} \otimes \mathbf{e}_{\Theta}+\lambda \mathbf{e}_{z} \otimes \mathbf{e}_{Z}
$$

admits a polar decomposition $\mathbf{F}=\mathbf{V R}$, with

$$
\mathbf{R}=\mathbf{e}_{r} \otimes \mathbf{e}_{R}+\mathbf{e}_{\theta} \otimes \mathbf{e}_{\Theta}+\mathbf{e}_{z} \otimes \mathbf{e}_{Z}
$$

and

$$
\mathbf{V}=\frac{d r}{d R} \mathbf{e}_{r} \otimes \mathbf{e}_{r}+\frac{B r}{R} \mathbf{e}_{\theta} \otimes \mathbf{e}_{\theta}+\lambda \mathbf{e}_{z} \otimes \mathbf{e}_{z} .
$$

Substitution from Eq. (5.4) in the stress response equation (2.5) gives the principal stresses as

$$
\begin{gathered}
T_{r r}=\mu\left(1-\frac{R}{\lambda B r}\left(\frac{d R}{d r}\right)^{3}\right), \quad T_{\theta \theta}=\mu\left(1-\frac{R^{3}}{\lambda B^{3} r^{3}} \frac{d R}{d r}\right), \\
T_{z z}=\mu\left(1-\frac{R}{\lambda^{3} B r} \frac{d R}{d r}\right) .
\end{gathered}
$$

The equations of equilibrium (2.7) reduce to the radial equation (4.6) and substitution from Eqs. (5.5) 1.2 in this equation leads to the nonlinear second-order ordinary differential equation

$$
3 R \frac{d R}{d r} \frac{d^{2} R}{d r^{2}}+\left(\frac{d R}{d r}\right)^{3}-\frac{R^{3}}{B^{2} r^{3}}=0
$$

which again does not involve the axial stretch. With the change of variable [13, 18]

$$
t=\frac{R}{r} \frac{d r}{d R}>0
$$

Eq. (5.6) reduces to the first-order equation

$$
3 r \frac{d t}{d r}=4-3 t-\frac{t^{3}}{B^{2}}
$$

The cubic expression on the right-hand side of this equation has one real positive zero $\alpha$, say, and a complex conjugate pair $\beta$ and $\bar{\beta}$, with

$$
\alpha+\beta+\bar{\beta}=0, \quad \beta \bar{\beta}-\alpha^{2}=3 B^{2}, \quad \alpha \beta \bar{\beta}=4 B^{2} .
$$

Thus, Eq. (5.8) may be written as

$$
3 B^{2} r \frac{d t}{d r}=(\alpha-t)\left(t^{2}+\alpha t+\beta \bar{\beta}\right)
$$


or

$$
\frac{1}{r} \frac{d r}{d t}=\frac{B^{2}}{B^{2}+\alpha^{2}}\left[\frac{1}{\alpha-t}+\frac{2 t+\alpha}{2\left(t^{2}+\alpha t+\beta \bar{\beta}\right)}+\frac{3 \alpha}{2 \gamma^{2}} \frac{1}{1+(t+\alpha / 2)^{2} / \gamma^{2}}\right],
$$

with

$$
\gamma^{2}=\beta \bar{\beta}-\frac{\alpha^{2}}{4}=3\left(B^{2}+\frac{\alpha^{2}}{4}\right)
$$

Integration of Eq. (5.11) gives

$$
r^{2\left(1+\alpha^{2} / B^{2}\right)}=A \frac{t^{2}+\alpha t+\beta \bar{\beta}}{(\alpha-t)^{2}} g(t) ; \quad g(t)=\exp \left(\frac{3 \alpha}{\gamma} \tan ^{-1} \frac{2 t+\alpha}{2 \gamma}\right)
$$

where $A>0$ is a constant of integration. Equations (5.7) and (5.10) give

$$
3 B^{2} R \frac{d t}{d R}=t(\alpha-t)\left(t^{2}+\alpha t+\beta \bar{\beta}\right)
$$

which leads to

$$
R^{4\left(1+\alpha^{2} / B^{2}\right)}=C \frac{t^{3\left(1+\alpha^{2} / B^{2}\right)} g(t)}{(\alpha-t)^{4 / \alpha}\left(t^{2}+\alpha t+\beta \bar{\beta}\right)^{\alpha^{2} / B^{2}}},
$$

with $C>0$. Equations (5.13) and (5.15) are a parametric representation of the radial deformation function $\hat{r}(R)$ in Eq. (5.1), with parameter $t$ taking values $0<t<\alpha$.

When $B=1$, the function on the right-hand side of Eq. (5.8) has a zero $\alpha=1$ and Eq. (5.9) and (5.12) give $\beta \bar{\beta}=4$ and $\gamma=\frac{\sqrt{15}}{2}$. Equations (5.13) and (5.15) reduce to

$$
r^{4}=A \frac{t^{2}+t+4}{(1-t)^{2}} g(t) ; \quad R^{8}=C \frac{t^{6} g(t)}{(1-t)^{4}\left(t^{2}+t+4\right)}
$$

with

$$
g(t)=\exp \left[\frac{6}{\sqrt{15}} \tan ^{-1} \frac{2 t+1}{\sqrt{15}}\right]
$$

which is the solution obtained by Chung, Horgan, and Abeyaratne [13].

6. Eversion of a cylindrical sector. A deformation

$$
r=\hat{r}(R), \quad \theta=\Theta, \quad z=-\lambda Z,
$$

with $\frac{d r}{d R}<0$ and $\lambda>0$, describes eversion of a cylindrical sector. The deformation gradient

$$
\mathbf{F}=\frac{d r}{d R} \mathbf{e}_{r} \otimes \mathbf{e}_{R}+\frac{r}{R} \mathbf{e}_{\theta} \otimes \mathbf{e}_{\Theta}-\lambda \mathbf{e}_{z} \otimes \mathbf{e}_{Z}
$$

admits a polar decomposition $\mathbf{F}=\mathbf{V R}$, with

$$
\mathbf{R}=-\mathbf{e}_{r} \otimes \mathbf{e}_{R}+\mathbf{e}_{\theta} \otimes \mathbf{e}_{\boldsymbol{\Theta}}-\mathbf{e}_{z} \otimes \mathbf{e}_{Z}
$$

and

$$
\mathbf{V}=-\frac{d r}{d R} \mathbf{e}_{r} \otimes \mathbf{e}_{r}+\frac{r}{R} \mathbf{e}_{\theta} \otimes \mathbf{e}_{\theta}+\lambda \mathbf{e}_{z} \otimes \mathbf{e}_{z}
$$


Substitution from Eq. (6.4) in the stress response equation (2.5) gives the principal stresses as

$$
\begin{gathered}
T_{r r}=\mu\left(1+\frac{R}{\lambda r}\left(\frac{d R}{d r}\right)^{3}\right), \quad T_{\theta \theta}=\mu\left(1+\frac{R^{3}}{\lambda r^{3}} \frac{d R}{d r}\right), \\
T_{z z}=\mu\left(1+\frac{R}{\lambda^{3} r} \frac{d R}{d r}\right) .
\end{gathered}
$$

The radial equation of equilibrium reduces to

$$
3 R \frac{d R}{d r} \frac{d^{2} R}{d r^{2}}+\left(\frac{d R}{d r}\right)^{3}-\frac{R^{3}}{r^{3}}=0,
$$

which is the same as Eq. (5.6), with $B=1$. The change of variable

$$
t=-\frac{R}{r} \frac{d r}{d R}>0
$$

reduces Eq. (6.6) to the first-order equation

$$
3 r \frac{d t}{d r}=-(t+1)\left(t^{2}-t+4\right),
$$

which may be integrated to give

$$
r^{4}=A \frac{t^{2}-t+4}{(t+1)^{2}} h(t) ; \quad h(t)=\exp \left(-\frac{6}{\sqrt{15}} \tan ^{-1} \frac{2 t-1}{\sqrt{15}}\right),
$$

where $A(>0)$ is a constant of integration. Equations (6.7) and (6.8) give

$$
3 R \frac{d t}{d R}=t(t+1)\left(t^{2}-t+4\right),
$$

which leads to

$$
R^{8}=B \frac{t^{6} h(t)}{(t+1)^{4}\left(t^{2}-t+4\right)} .
$$

Equations (6.9) and (6.11) are a parametric representation of the solution for cylindrical eversion, with parameter $t>0$. This solution is simply that obtained by Chung, Horgan, and Abeyaratne [13], with $t$ replaced by $-t$.

7. Eversion of a spherical sector. A deformation

$$
r=\hat{r}(R), \quad \theta=\pi-\Theta, \quad \phi=\Phi,
$$

with $\frac{d r}{d R}<0$, describes eversion of a spherical sector. The deformation gradient

$$
\mathbf{F}=\frac{d r}{d R} \mathbf{e}_{r} \otimes \mathbf{e}_{R}-\frac{r}{R}\left(\mathbf{e}_{\theta} \otimes \mathbf{e}_{\boldsymbol{\Theta}}-\mathbf{e}_{\phi} \otimes \mathbf{e}_{\Phi}\right)
$$

admits a polar decomposition $\mathbf{F}=\mathbf{V R}$, with

$$
\mathbf{R}=-\mathbf{e}_{r} \otimes \mathbf{e}_{R}-\mathbf{e}_{\theta} \otimes \mathbf{e}_{\Theta}+\mathbf{e}_{i,} \otimes \mathbf{e}_{\Phi}
$$

and

$$
\mathbf{V}=-\frac{d r}{d R} \mathbf{e}_{r} \otimes \mathbf{e}_{r}+\frac{r}{R}\left(\mathbf{e}_{\theta} \otimes \mathbf{e}_{\theta}+\mathbf{e}_{i j} \otimes \mathbf{e}_{i j}\right)
$$


Substitution from Eq. (7.4) in the stress response equation (2.5) gives the principal stresses as

$$
T_{r r}=\mu\left(1+\frac{R^{2}}{r^{2}}\left(\frac{d R}{d r}\right)^{3}\right), \quad T_{\theta \theta}=T_{\phi \phi}=\mu\left(1+\frac{R^{4}}{r^{4}} \frac{d R}{d r}\right) .
$$

The equations of equilibrium (2.8) reduce to the radial equation

$$
\frac{d T_{r r}}{d r}+\frac{2}{r}\left(T_{r r}-T_{\theta \theta}\right)=0,
$$

or

$$
3 r^{3} R^{2} \frac{d R}{d r} \frac{d^{2} R}{d r^{2}}+2 r^{3} R\left(\frac{d R}{d r}\right)^{3}-2 R^{4}=0,
$$

which is the same as the equation obtained by Chung, Horgan, and Abeyaratne [13] in the case of radial expansion or compaction $\left(\frac{d r}{d R}>0\right)$.

The change of variable

$$
t=-\frac{R}{r} \frac{d r}{d R}>0
$$

leads to the first-order equation

$$
3 r \frac{d t}{d r}=-(t+1)\left(2 t^{2}-2 t+5\right) .
$$

Proceeding as in the previous section leads to a parametric representation of the deformation, with parameter $t>0$, which is obtained from the solution in [13] by replacing $t$ by $-t$, viz.

$$
r^{6}=A \frac{2 t^{2}-2 t+5}{(t+1)^{2}} k(t), \quad R^{15}=B \frac{t^{9} k(t)}{(t+1)^{5}\left(2 t^{2}-2 t+5\right)^{2}}
$$

with

$$
k(t)=\exp \left(-2 \tan ^{-1} \frac{2 t-1}{3}\right) .
$$

8. Torsion and extension of a circular cylinder or tube. The deformation

$$
r=\hat{r}(R), \quad \theta=\Theta+D Z, \quad z=\lambda Z,
$$

with $\frac{d r}{d R}>0, D>0$, and $\lambda>0$, describes torsion of a cylinder, with twist $D$ per unit undeformed length, accompanied by uniform axial stretch and by radial deformation. The deformation gradient tensor is

$$
\mathbf{F}=\frac{d r}{d R} \mathbf{e}_{r} \otimes \mathbf{e}_{R}+\frac{r}{R} \mathbf{e}_{\theta} \otimes \mathbf{e}_{\Theta}+\lambda \mathbf{e}_{z} \otimes \mathbf{e}_{Z}+D r \mathbf{e}_{\theta} \otimes \mathbf{e}_{Z} .
$$

A routine calculation gives the physical components of $\mathbf{B}^{-1}=\left(\mathbf{F}^{-1}\right)^{\mathrm{T}} \mathbf{F}^{-1}$ in the $(r, \theta, z)$ system as

$$
\mathbf{B}^{-1}=\left[\begin{array}{ccc}
\left(\frac{d R}{d r}\right)^{2} & 0 & 0 \\
0 & \frac{R^{2}}{r^{2}} & -\frac{D R^{2}}{\lambda r} \\
0 & -\frac{D R^{2}}{\grave{\lambda} r} & \frac{1+D^{2} R^{2}}{\lambda^{2}}
\end{array}\right] .
$$


Substitution from Eqs. (8.2) and (8.3) in the stress response equation (2.5) gives the physical components of Cauchy stress as

$$
\begin{gathered}
T_{r r}=\mu\left(1-\frac{R}{\lambda r}\left(\frac{d R}{d r}\right)^{3}\right), \quad T_{\theta z}=\frac{\mu D R^{3}}{\lambda^{2} r^{2}} \frac{d R}{d r}, \\
T_{\theta \theta}=\mu\left(1-\frac{R^{3}}{\lambda r^{3}} \frac{d R}{d r}\right), \quad T_{z r}=0, \\
T_{z z}=\mu\left(1-\frac{R\left(1+D^{2} R^{2}\right)}{\lambda^{3} r} \frac{d R}{d r}\right), \quad T_{r \theta}=0 .
\end{gathered}
$$

Since these depend on the radial coordinate $r$ only, it follows that only the in-plane stresses $T_{r r}$ and $T_{\theta \theta}$ enter in the equations of equilibrium. Furthermore, these stresses are unaffected by the torsion (for a Blatz-Ko material), i.e., they are the same as those in Eq. (5.5) 1,2 , with $B=1$. It follows that a material with strain energy (2.4) admits a deformation of form (8.1) with the radial deformation given by Eqs. (5.16) and (5.17).

In particular, it follows that Blatz-Ko materials admit pure torsion, or torsion superposed on homogeneous axisymmetric stretch

$$
r=\alpha R, \quad \theta=\Theta+D Z, \quad z=\lambda Z .
$$

This has also been observed by Beatty [15]. The stress components are given by (8.4) and $(8.5)$ as

$$
\begin{gathered}
T_{r r}=\mu\left(1-\frac{1}{\lambda \alpha^{4}}\right), \quad T_{\theta z}=\frac{\mu D R}{\lambda^{2} \alpha^{3}}, \\
T_{\theta \theta}=\mu\left(1-\frac{1}{\lambda \alpha^{4}}\right), \quad T_{z r}=0, \\
T_{z z}=\mu\left(1-\frac{1+D^{2} R^{2}}{\lambda^{3} \alpha^{2}}\right), \quad T_{r \theta}=0 .
\end{gathered}
$$

If the lateral stretch $\alpha$ is given by

$$
\alpha=\lambda^{-1 / 4}
$$

then the stress components $(8.6)$ reduce to

$$
\begin{gathered}
T_{z z}=\mu\left(1-\lambda^{-5 / 2}\left(1+D^{2} R^{2}\right)\right), \quad T_{\theta z}=\frac{\mu D R}{\lambda^{5 / 4}}, \\
T_{r r}=T_{\theta \theta}=T_{z r}=T_{r \theta}=0,
\end{gathered}
$$

and so the lateral surface of a solid circular cylinder or a hollow tube can be rendered traction-free when (8.7) holds. The resultant normal force $T$ on the ends of a hollow cylinder of inner and outer radii $R_{1}$ and $R_{2}$ is given by Eq. (8.8), as

$$
T=2 \pi \int_{r \alpha R_{1}}^{\alpha R_{2}} T_{z z} r d r=\frac{1}{2} \pi \mu\left(R_{2}^{2}-R_{1}^{2}\right)\left(2\left(\lambda^{-1 / 2}-\lambda^{-3}\right)-\lambda^{-3} D^{2}\left(R_{1}^{2}+R_{2}^{2}\right)\right) .
$$

In particular, the normal force needed to support pure torsion $(\lambda=1)$ is a compressive force

$$
T=-\frac{1}{2} \pi \mu D^{2}\left(R_{2}^{4}-R_{1}^{4}\right) .
$$


The relationship between the amount of twist and the axial stretch, when there is no resultant axial force, is obtained by setting $T=0$ in Eq. (8.9), giving

$$
\lambda^{5 / 2}=1+\frac{1}{2} D^{2}\left(R_{1}^{2}+R_{2}^{2}\right) \text {. }
$$

The twisting moment is given as

$$
M=2 \pi \int_{\alpha R_{1}}^{\alpha R_{2}} T_{\theta z} r^{2} d r=\frac{\pi \mu D}{2 \lambda^{2}}\left(R_{2}^{4}-R_{1}^{4}\right) .
$$

Thus, if the axial stretch $\lambda$ is specified then the torsional rigidity $\frac{M}{D}$ is constant. Furthermore, when $\lambda=1,(8.10)$ and $(8.12)$ yield the result

$$
|T|=D M,
$$

a universal relation between applied twisting moment and compressive thrust. The result (8.13) was also obtained by Beatty [15]. On the other hand, if there is no resultant normal force on the ends, then elimination of $\lambda$ from Eqs. (8.11) and (8.12) gives a nonlinear relationship between $M$ and $D$.

9. Bending and shearing deformations. The result obtained in the previous section suggests that it may be worthwhile to examine, for the Blatz-Ko strain energy (2.4), nonisochoric generalizations of the five families of nonhomogeneous deformations which are known to be controllable deformations for all homogeneous, isotropic, incompressible elastic solids [1,3].

Family 1.

$$
x=x(R), \quad y=C \Theta+D Z, \quad z=E \Theta+F Z,
$$

with

$$
(C F-D E) \frac{d x}{d R}>0 .
$$

This deformation describes straightening and stretching of a cylindrical sector, together with shearings in the $y$ - and $z$-directions. A routine calculation gives the Cartesian components of the deformation tensor $\mathbf{B}^{-1}$ as

$$
\mathbf{B}^{-1}=\frac{1}{(C F-D E)^{2}}\left[\begin{array}{ccc}
(C F-D E)^{2}\left(\frac{d R}{d x}\right)^{2} & 0 & 0 \\
0 & F^{2} R^{2}+E^{2} & -F D R^{2}-C E \\
0 & -F D R^{2}-C E & C^{2}+D^{2} R^{2}
\end{array}\right] .
$$

The Cauchy stress is obtained by substituting from Eq. (9.3) in the stress response equation (2.5). The equation of equilibrium again reduces to the form (3.7), leading to an equation of form (3.9) with $\lambda B$ replaced by $C F-D E$. Thus, the deformation (9.1) takes the form

$$
x=\frac{3}{4}(C F-D E)^{1 / 3} R^{4 / 3}, \quad y=C \Theta+D Z, \quad z=E \Theta+F Z .
$$

Family 2.

$$
r=\hat{r}(X), \quad \theta=C Y+D Z, \quad z=E Y+F Z,
$$


with

$$
(C F-D E) \frac{d r}{d X}>0 .
$$

This deformation describes bending and stretching of a rectangular block, together with torsional and axial shearings. A routine calculation gives the physical components of $\mathbf{B}^{-1}$ in the cylindrical system as

$$
\mathbf{B}^{-1}=\frac{1}{(C F-D E)^{2}}\left[\begin{array}{ccc}
(C F-D E)^{2}\left(\frac{d X}{d r}\right)^{2} & 0 & 0 \\
0 & \frac{E^{2}+F^{2}}{r^{2}} & -\frac{C E+D F}{r} \\
0 & -\frac{C E+D F}{r} & C^{2}+D^{2}
\end{array}\right],
$$

and the stress is obtained by substituting from this equation in Eq. (2.5). The equations of equilibrium again reduce to the radial equation (4.6), and the stress components $T_{r r}$ and $T_{\theta \theta}$ are those in Eqs. (4.5) 1,2 , with

$$
B=\frac{C F-D E}{\sqrt{E^{2}+F^{2}}}, \quad \lambda=\sqrt{E^{2}+F^{2}} .
$$

The deformation is therefore given by Eqs. (4.9), (9.5), and (9.8).

Family 3.

$$
r=\hat{r}(R), \quad \theta=C \Theta+D Z, \quad z=E \Theta+F Z,
$$

with

$$
(C F-D E) \frac{d r}{d R}>0 .
$$

This deformation may describe extension and radial expansion or compaction of cylinders, eversion or bending of cylindrical sectors, and torsional and axial shearing. A routine calculation gives the physical components of $\mathbf{B}^{-1}$ in the $(r, \theta, z)$ system as

$$
\mathbf{B}^{-1}=\frac{1}{(C F-D E)^{2}}\left[\begin{array}{ccc}
(C F-D E)^{2}\left(\frac{d R}{d r}\right)^{2} & 0 & 0 \\
0 & \frac{F^{2} R^{2}+E^{2}}{r^{2}} & -\frac{F D R^{2}+C E}{r} \\
0 & -\frac{F D R^{2}+C E}{r} & C^{2}+D^{2} R^{2}
\end{array}\right] .
$$

Substitution from this equation in the stress response equation (2.5) gives the physical components of the Cauchy stress tensor. In particular, the components $T_{r r}$ and $T_{\theta \theta}$, which are the only components to appear in the equation of equilibrium (4.6), are given as

$$
T_{r r}=\mu\left(1-\frac{R}{r(C F-D E)}\left(\frac{d R}{d r}\right)^{3}\right), \quad T_{\theta \theta}=\mu\left(1-\frac{R\left(F^{2} R^{2}+E^{2}\right)}{r^{3}(C F-D E)^{3}} \frac{d R}{d r}\right) .
$$

Thus, the equation of equilibrium reduces to

$$
3 R \frac{d R}{d r} \frac{d^{2} R}{d r^{2}}+\left(\frac{d R}{d r}\right)^{3}-\frac{\left(F^{2} R^{2}+E^{2}\right) R}{(C F-D E)^{2} r^{3}}=0
$$


This equation may be reduced to a first-order equation by a change of variable (5.7) or (6.7) only if $E=0$, i.e., when there is no axial shearing. In this case, the deformation is given by Eqs. (5.13) and (5.15), with $B=C$, for $\frac{d r}{d R}>0$, and the solution for eversion and torsional shearing is obtained by substituting $-t$ for $t$.

Family 4. The controllable isochoric deformations in Family 4 describe spherically radial expansion or compaction, or eversion. These have been examined for the Blatz-Ko strain energy function, by Chung, Horgan, and Abeyaratne [13], and in Sec. 7.

Family 5. A deformation of form

$$
r=\hat{r}(R), \quad \theta=B \Theta+f(R), \quad z=\lambda Z,
$$

describes radial deformation, bending, azimuthal shearing and uniform axial stretching of a cylindrical sector. This deformation may also be written in inverse form as

$$
R=\widetilde{R}(r), \quad \Theta=\frac{1}{B}(\theta-F(r)), \quad Z=\frac{z}{\lambda} .
$$

A routine calculation gives the physical components of $\mathbf{B}^{-1}$ in the $(r, \theta, z)$ system as

$$
\mathbf{B}^{-1}=\left[\begin{array}{ccc}
\left.\left(\frac{d R}{d r}\right)^{2}+\frac{R^{2}}{B^{2}} \frac{d F}{d r}\right)^{2} & -\frac{R^{2}}{B^{2} r} \frac{d F}{d r} & 0 \\
-\frac{R^{2}}{B^{2} r} \frac{d F}{d r} & \frac{R^{2}}{B^{2} r^{2}} & 0 \\
0 & 0 & \frac{1}{\lambda^{2}}
\end{array}\right] .
$$

Equations (2.5) and (9.16) give the Cauchy stress components as

$$
\begin{gathered}
T_{r r}=\mu\left(1-\frac{R}{\lambda B r} \frac{d R}{d r}\left[\left(\frac{d R}{d r}\right)^{2}+\frac{R^{2}}{B^{2}}\left(\frac{d F}{d r}\right)^{2}\right]\right), \quad T_{\theta z}=0, \\
T_{\theta \theta}=\mu\left(1-\frac{R^{3}}{\lambda B^{3} r^{3}} \frac{d R}{d r}\right), \quad T_{z r}=0, \\
T_{z z}=\mu\left(1-\frac{R}{\lambda^{3} B r} \frac{d R}{d r}\right), \quad T_{r \theta}=\frac{\mu R^{3}}{\lambda B^{3} r^{2}} \frac{d R}{d r} \frac{d F}{d r} .
\end{gathered}
$$

The equations of equilibrium (2.7) reduce to Eq. (4.6) and

$$
\frac{d T_{\theta r}}{d r}+\frac{2}{r} T_{\theta r}=0
$$

and substitution from Eq. $(9.17)_{6}$ leads to

$$
\frac{d R}{d r} \frac{d F}{d r}=\frac{A^{3}}{R}
$$

where $A$ is a constant of integration. Substitution from Eqs. (9.17) 1,2 and (9.19) in the radial equation of equilibrium gives a nonlinear second-order ordinary differential equation for the function $\widetilde{R}(r)$, so that Blatz-Ko materials can sustain deformations of the form (9.14). However, this equation is reduced to a first-order equation by the change of variable (5.7) only if $A=0$, in which case the deformation (9.14) differs from (5.1) only by a rigid rotation. 
Acknowledgments. The work of M. M. Carroll was supported by a contribution from the Shell Companies Foundation, in support of a Shell Distinguished Chair at the University of California, Berkeley, while that of C. O. Horgan was supported by the National Science Foundation under Grant No. MSM-89-04719 and by the U. S. Air Force Office of Scientific Research under Grant No. AFOSR-89-0470.

\section{REFERENCES}

[1] J. L. Ericksen, Deformations possible in every isotropic, incompressible, perfectly elastic body. Z. Angew. Math. Phys. 5, 466-489 (1954)

[2] J. L. Ericksen, Deformations possible in every compressible, isotropic, perfectly elastic material, J. Math. and Phys. 34, 126-128 (1955)

[3] M. Singh and A. C. Pipkin, Note on Ericksen's problem, Z. Angew. Math. Phys. 16, 706-709 (1965)

[4] P. K. Currie and M. Hayes, On non-universal finite elastic deformations, Finite Elasticity, Proceedings of IUTAM Symposium (D. E. Carlson and R. T. Shield, eds.), Martinus Nijhoff, The Hague, 1982, pp. 143-150

[5] F. John, Plane strain problems for a perfectly elastic material of harmonic type, Comm. Pure Appl. Math. 13, 239-296 (1960)

[6] R. W. Ogden and D. A. Isherwood, Solution of some finite plane-strain problems for compressible elastic solids, Quart. J. Mech. Appl. Math. 31, 219-249 (1978)

[7] E. Varley and E. Cumberbatch, Finite deformations of elastic materials surrounding cylindrical holes, J. Elasticity 10, 341-405 (1980)

[8] C. H. Wu, Plane-strain buckling of a crack in a harmonic solid subjected to crack-parallel compression, J. Appl. Mech. 46, 597-604 (1979)

[9] A. H. Jafari, R. Abeyaratne, and C. O. Horgan, The finite deformation of a pressurized circular tube for a class of compressible materials, Z. Angew. Math. Phys. 35, 227-246 (1984)

[10] R. W. Ogden, Non-linear Elastic Deformations, Ellis Horwood, Chichester, 1984

[11] R. Abeyaratne and C. O. Horgan, The pressurized hollow sphere problem in finite elastostatics for a class of compressible materials, Int. J. Solids Struct. 20. 715-723 (1984)

[12] L. T. Wheeler, Finite deformation of a harmonic elastic medium containing an ellipsoidal cavity, Internat. J. Solids Struct. 21, 799-804 (1985)

[13] D. T. Chung, C. O. Horgan, and R. Abeyaratne, The finite deformation of internally pressurized hollow cylinders and spheres for a class of compressible elastic materials, Internat. J. Solids Struct. 22, 1557-1570 (1986)

[14] P. J. Blatz and W. L. Ko, Application of finite elasticity to the deformation of rubbery materials, Trans. Soc. Rheol. 6, 223-251 (1962)

[15] M. F. Beatty, Topics in finite elasticity: hyperelasticity of rubber, elastomers, and biological tissueswith examples, Appl. Mech. Reviews 40, 1699-1734 (1987)

[16] M. M. Carroll, Finite strain solutions in compressible isotropic elasticity, J. Elasticity 20, 65-92 (1988)

[17] D. M. Haughton, Inflation of thick-walled compressible elastic spherical shells, IMA J. Appl. Math. 39, 259-272 (1987)

[18] C. O. Horgan, Some remarks on axisymmetric solutions in finite elastostatics for compressible materials, Proc. Roy. Irish Acad. Sect. A 89, 185-193 (1989)

[19] J. K. Knowles and E. Sternberg, On the ellipticity of the equations of nonlinear elastostatics for a special material, J. Elasticity 5, 341-361 (1975) 